Andrew Diniz da Costa

Automação do Processo de Gerência do Teste de Software

Tese de Doutorado

Tese apresentada como requisito parcial para obtenção do grau de Doutor pelo Programa de Pós-graduação em Informática do Departamento de Informática do Centro Técnico Científico da PUC-Rio.

Orientador: Prof. Carlos José Pereira de Lucena

Rio de Janeiro

Agosto de 2012 


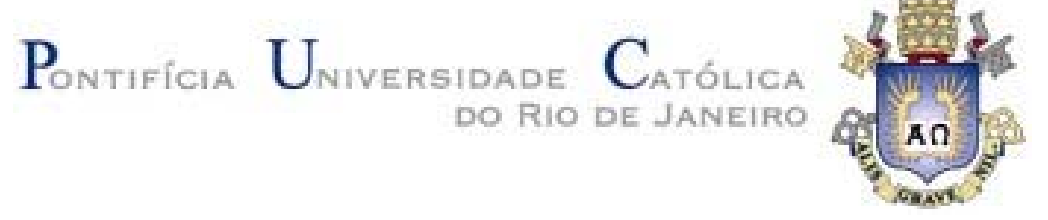

Andrew Diniz da Costa

\section{Automação do Processo de Gerência do \\ Teste de Software}

Tese apresentada como requisito parcial para obtenção do grau de Doutor pelo Programa de Pós-graduação em Informática do Departamento de Informática do Centro Técnico Científico da PUC-Rio. Aprovada pela Comissão Examinadora abaixo assinada.

Prof. Carlos José Pereira de Lucena
Orientador
Departamento de Informática - PUC-Rio

Prof. Alessandro Garcia

Departamento de Informática - PUC-Rio

Prof. Arndt Von Staa

Departamento de Informática - PUC-Rio

Prof. Viviane Torres da Silva

Universidade Federal Fluminense - UFF

Prof. Roberta de Souza Coelho Universidade Federal do Rio Grande do Norte - UFRN

Prof. José Eugenio Leal Coordenador Setorial do Centro Técnico Científico - PUC-Rio

Rio de Janeiro, 10 de agosto de 2012 
Todos os direitos reservados. É proibida a reprodução total ou parcial do trabalho sem autorização da universidade, do autor e do orientador.

\section{Andrew Diniz da Costa}

Possui formação técnica no Instituto Brasileiro de Pesquisa em Informática (IBPI). Graduou-se em Bacharelado em Informática na PUC-Rio em 2006. Recebeu o título de Mestre em Informática na PUC-Rio em 2008. É IBM Certified Solution Designer -Rational Functional Tester for Java e possui o título de "Certified Tester Foundation Level - CTFL" pela "Brazilian Software Testing Qualifications Board - BSTQB". Desde 2003 trabalha no Laboratório de Engenharia de Software (LES) tanto em projetos de pesquisa como em projetos industriais. Atualmente é líder de três equipes de teste no LES compostas por doze pessoas. Possui mais de 10 anos de experiência em desenvolvimento de software a partir de diferentes linguagens de programação.

Ficha Catalográfica

Costa, Andrew Diniz da

Automação do processo de Gerência do Teste de Software / Andrew Diniz da Costa ; orientador: Carlos José Pereira de Lucena. - 2012.

$171 \mathrm{f}$; $30 \mathrm{~cm}$

Tese (doutorado) - Pontifícia Universidade Católica do Rio de Janeiro, Rio de Janeiro, 2012.

Inclui bibliografia.

1. Informática - Tese. 2. Coordenação de Testes de Software.. 3. Agentes de Software Autoadaptativos . 4. Autoteste. 5. Modelagem de Teste. I. Lucena, Carlos José Pereira de. II. Pontifícia Universidade Católica do Rio de Janeiro. Departamento de Informática. 
A Deus, a minha mãe e ao meu pai que me ensinaram a acreditar que tudo é possível. 


\section{Agradecimentos}

A minha mãe Nazaré e ao meu pai Wagner que são meus ídolos. Obrigado pelo exemplo de pais, por todo esforço, carinho, confiança, investimento, paciência e amor concedido durante toda minha vida. Obrigado pelas conversas e apoio para fazer o doutorado. Vocês tiveram uma grande parcela de importância nessas conquistas obtidas ao passar da minha vida. Serei eternamente grato. Amo muito vocês!! =)

A toda minha família do Pará e do Amapá que mesmo afastados sempre torceram por mim.

Ao professor Carlos José Pereira de Lucena pelas orientações, exemplo de profissional, oportunidades e confiança depositada durante minha graduação, mestrado e doutorado.

A professora Viviane Torres da Silva pelas enormes contribuições durante todos esses anos de trabalho em conjunto. 
Aos professores Alessandro Garcia, Arndt Von Staa, e Roberta Coelho que tiveram contribuições importantes para a tese de doutorado.

A Paola, pelas conversas, apoio, momentos de descontração e amizade oferecidos durante esses quatro anos de doutorado.

A todos os amigos do LES, em especial ao grupo de testes de software que trabalhou dia a dia comigo em diferentes projetos, transformando os dias mais divertidos e descontraídos.

Ao CNPq pelo apoio financeiro da bolsa de doutorado.

E principalmente a Deus por tudo.

Muito obrigado!! 


\section{Resumo}

Costa, Andrew Diniz da; Lucena, Carlos José Pereira de. Automação do

Processo de Gerência do Teste de Software. Rio de Janeiro, 2012. 171p. Tese de Doutorado - Departamento de Informática, Pontifícia Universidade Católica do Rio de Janeiro.

Teste de software é uma atividade crítica no processo de desenvolvimento de sistemas, principalmente devido ao aumento da complexidade das aplicações atuais e pelo impacto que tais aplicações podem gerar. Relacionado a essa complexidade, o paradigma de sistemas multiagentes tem sido utilizado especialmente quando entidades pró-ativas, autônomas, autoadaptáveis e distribuídas precisam ser desenvolvidas. Para acompanhar a criação, manutenção e execução dos testes nesses sistemas, torna-se necessária a adoção de um processo de gerência, pois prevê a realização das atividades de planejamento, projeto, execução e acompanhamento dos testes. Visando ajudar nessa gerência, a tese apresenta o Java self-Adaptive Agent Framework for SelfTest $(\mathrm{JAAF}+\mathrm{T})$, framework que permite a criação de agentes autoadaptativos capazes de realizar autoteste, isto é, coordenar a execução dos testes necessários para validar suas autoadaptações. Como diversas informações são usadas para ajudar na gerência desses testes, documentá-las ajuda a entender como evoluir e executá-los. Baseada nessa preocupação, a tese oferece uma nova linguagem de modelagem chamada de UML Testing Profile for Coordination (UTP-C), perfil (profile) da UML que permite a modelagem dessas informações. Por fim, para automatizar o processo de gerência dos testes executados por agentes autoadaptativos, a tese apresenta ferramentas capazes de gerar de forma automática artefatos usados pelo JAAF+T baseados em modelos UTP-C.

\section{Palavras-chave}

Coordenação de testes de software; agentes de software autoadaptativos; autoteste; modelagem de teste. 


\section{Abstract}

Costa, Andrew Diniz da; Lucena, Carlos José Pereira de. Automation of the Management Process of the Test of Software. Rio de Janeiro, 2012. 171p. DSc Thesis - Departamento de Informática, Pontifícia Universidade Católica do Rio de Janeiro.

Testing software systems has become a critical activity of software development over time. Especially when the development of complex systems, which are able to self-adapt theirs behaviors when necessary, is becoming extremely common. In this context, the multi-agent system (MAS) paradigm has been used especially when distributed, autonomous and pro-active entities are represented. Aiming to control the creation, maintenance and execution of tests on these systems, it is necessary to adopt a management process that considers the activities of planning, design, execution and monitoring of the tests. In order to help this management, the thesis presents the Java self-Adaptive Agent Framework for Self-Test $(\mathrm{JAAF}+\mathrm{T})$, that aims to allow the creation of selfadaptive agents that perform self-tests. Since several data are used to help the management of the tests, documenting them helps to understand how to evolve and execute them. Based on this concern, the thesis offers a new modeling language called UML Testing Profile for Coordination (UTP-C), profile of the UML that allows the modeling of these data. At last, but not least important, tools, which were created to automate the management of the tests executed for selfadaptive agents, are presented to generate useful artifacts used for instances of the JAAF+T based on UTP-C models.

\section{Keywords}

Coordination of software testing; self-adaptive agents of software; selftesting; test modeling. 


\section{Sumário}

1 Introdução 16

$\begin{array}{ll}\text { 1.1. Motivação } & 17\end{array}$

1.2. O Problema 19

1.3. Principais Contribuições 19

1.4. Organização do Documento 20

2 Fundamentação Teórica $\quad 21$

2.1. Conceitos Gerais da Área de Testes de Software 21

2.2. UML Testing Profile (UTP) 24

2.3. Sistemas Multiagentes 26

2.4. Sistemas Autoadaptativos que Realizam Autoteste 28

2.5. Framework JADE $\quad 29$

3 Informações para Coordenação da Execução de Testes 32

3.1. Procedimentos Empíricos 33

3.2. Informações Identificadas $\quad 34$

3.3. Considerações Finais 37

4 JAAF+T: Framework de Autoteste para Agentes de Software 38

4.1. Visão Geral do JAAF+T 39

4.2. Arquivos $X M L$

4.3. Pontos Fixos e Flexíveis 51

4.4. Como Usar o Framework JAAF+T 53

4.5. Considerações Finais $\quad 54$

5 Modelo Conceitual de Teste 56

5.1. Caso de Teste $\quad 57$

5.2. Teste 58

5.3. Artefato em Teste $\quad 59$

5.4. Suite de Teste $\quad 59$

5.5. Classificação de Teste 60

5.6. Pacote de Desenvolvimento 61

5.7. Sistema em Teste 61

5.8. Critério de Seleção de Testes 61 
5.9. Semântica de Dependências de Testes 62

5.10. Possíveis Perguntas para o Modelo Conceitual 62

5.11. Considerações Finais 63

6 Linguagem de Modelagem UTP-C 64

6.1. Os Mecanismos de Extensão da UML e UTP 64

6.2. Metamodelo da UML Testing Profile for Coordination (UTP-C) 65

6.2.1. Suite de Teste 68

6.2.2. Contexto de Teste (TestContext) 69

$\begin{array}{ll}\text { 6.2.3. Caso de Teste } & 71\end{array}$

6.2.4. Critério de Seleção de Teste 75

$\begin{array}{ll}\text { 6.2.5. Classificação de Teste } & 77\end{array}$

6.2.6. Pacote de Desenvolvimento 79

6.2.7. Artefato em Teste $\quad 80$

6.2.8. Estereótipos para Relacionamentos de Dependência 82

6.3. Mapeamento do Modelo Conceitual para a Linguagem UTP-C 86

6.4. Avaliações Empíricas para a UTP-C 88

6.4.1. Avaliação para Manutenção de Modelos 89

6.4.2. Avaliação para Criação de Modelos 94

6.4.3. Resultados das Avaliações Aplicadas 96

$\begin{array}{ll}\text { 6.5. Considerações Finais } & 100\end{array}$

7 Estudo de Caso Usando UTP-C com JAAF+T 101

$\begin{array}{ll}\text { 7.1. Abordagem Adotada } & 101\end{array}$

$\begin{array}{ll}\text { 7.1.1. Diagramas Estáticos UTP-C } & 101\end{array}$

7.1.2. Diagramas Dinâmicos UTP-C 102

7.1.3. Processo de Adaptação no JAAF+T 103

$\begin{array}{ll}\text { 7.1.4. Testes no JAAF+T } & 103\end{array}$

7.1.5. Dados de Entrada e Saída para Testes no JAAF+T 103

7.1.6. Fluxos de Execução dos Testes no JAAF+T 104

7.1.7. Critérios de Seleção dos Testes no JAAF+T 104

7.2. O Exemplo do Mercado Virtual 105

$\begin{array}{ll}\text { 7.2.1. Modelagem a partir da UTP-C } & 107\end{array}$

7.2.2. Autoadaptação Realizada pelo Agente Comprador 112

7.3. Outros Exemplos Usando UTP-C e JAAF+T 119

$\begin{array}{ll}\text { 7.4. Discussão } & 121\end{array}$

$\begin{array}{ll}\text { 7.5. Considerações Finais } & 124\end{array}$ 
8 Ferramentas para Aplicar Testes Baseados em Modelos

8.1. Mapeamento entre Arquivos XML do JAAF+T e Modelos UTP-C 125

8.2. Componentes LUA 128

8.3. Plug-in para o RSA 130

8.3.1. Visão Geral da Arquitetura do Plug-in 131

8.3.2. Geração de Relatórios e Comentários Javadoc 133

8.3.3. Geração dos arquivos TF.xml e CFF.xml 138

8.3.4. Avaliação da Ferramenta de Teste 139

8.4. Discussão 144

8.5. Considerações Finais 145

9 Trabalhos Relacionados $\quad 146$

9.1. Trabalhos Voltados a Autoadaptação e Autoteste 146

9.2. Linguagens e Ferramentas de Gerenciamento de Teste 147

10 Conclusões e Trabalhos Futuros 151

10.1. Principais Vantagens das Abordagens Propostas 151

10.2. Principais Limitações das Abordagens Propostas 152

10.3. Trabalhos Futuros 153

$\begin{array}{ll}\text { Referências } & 155\end{array}$

Apêndice A. Exemplo de Questionário Aplicado para Avaliação de Manutenção de Modelos baseados na UTP-C 164

Apêndice B. Modelos UTP-C do Estudo de Caso Mercado Virtual Criados no RSA

Apêndice C. XMLs gerados pelo Plug-in para o RSA Referente ao Domínio Mercado Virtual 


\section{Lista de Figuras}

Figura 1. Processo de autoadaptação proposto pela IBM 29

Figura 2: Plataformas de agentes do JADE $\quad 30$

Figura 3. Diagrama de classes do JADE 31

Figura 4. Procedimentos empíricos para identificar informações para

coordenação de teste a partir da execução de agentes de software. 33

Figura 5. Control-loop provido pelo JAAF+T. 41

Figura 6. Diagrama de classe do JAAF+T. 42

Figura 7. Esquema do arquivo TF.xml. 45

Figura 8. Esquema do arquivo DF.xml. $\quad 46$

$\begin{array}{ll}\text { Figura 9. Esquema do arquivo CFF.xml. } & 48\end{array}$

Figura 10. Esquema do arquivo CEF.xml. 49

Figura 11. Modelo detalhando relacionamentos entre informações de teste. $\quad 57$

Figura 12. Metamodelo da UML com estereótipos da UTP-C. 66

Figura 13. Estereótipos que expressam semântica de dependências. 67

Figura 14. OrderedSuite na UTP-C. 68

Figura 15. Exemplo de modelagem usando <<OrderedSuite >>. 69

Figura 16. TestContext na UTP-C. $\quad 69$

Figura 17. Exemplo de Test Context. $\quad 70$

Figura 18. Exemplo de diagrama de atividades com test contexts. 71

Figura 19. Caso de Teste na UTP-C. 71

Figura 20. Exemplo de um caso de teste de unidade modelado. 73

Figura 21. Exemplo de casos de teste de aceitação modelados. 73

Figura 22. Exemplo de diagrama de atividades com casos de teste. 74

Figura 23. Critério de seleção na UTP-C. 75

Figura 24. Exemplo de modelagem de um critério de seleção da UTP-C. $\quad 76$

Figura 25. Classificação de Teste na UTP-C. $\quad 77$

Figura 26. Modelagem de classificações de teste. 78

Figura 27. Pacote de Desenvolvimento na UTP-C. 79

Figura 28. Modelagem usando o estereótipo <<Development >>. 79

Figura 29. Estereótipo relacionado a artefatos de um sistema. 80

Figura 30. Modelando artefatos usados em sistemas. 82

Figura 31. Semântica de dependências incluídas. 83 
Figura 32. Dependência entre testes de criação de artefato.

Figura 33. Dependência entre testes informando o caso de teste que depende.

Figura 34. Procedimentos empíricos adotados na avaliação de manutenção. 94

Figura 35. Resultados da avaliação de manutenção de modelos. $\quad 97$

Figura 36. Resultados da avaliação de criação de modelos. 99

Figura 37. Visão geral da reputação de testemunho. 106

Figura 39. Modelagem de classificação de teste no mercado virtual. 109

Figura 40. Critérios do mercado virtual, modelados a partir da UTP-C. $\quad 110$

Figura 41. Suite do mercado virtual modelada em um diagrama de classes. 111

Figura 42. Diagrama de atividades do suíte do sistema mercado virtual. 112

Figura 43. Transformação realizada pelo componente RSATF.lua. 129

Figura 44. Transformação realizada pelo componente RSACFF.lua. 129

Figura 45. Modelo conceitual do plug-in para o RSA. 131

Figura 46. Diagrama de classes do novo plug-in para o RSA. 132

Figura 47. Diagrama de classes e atividades da UTP-C modeladas no RSA. 133

Figura 48. Atributos adicionais de um caso de teste modelado. 134

Figura 49. Relatório gerado a partir do plug-in. 135

Figura 50. Tela para geração de artefatos de teste. 136

Figura 51. Diagrama de classe UTP-C modelado no RSA para o sistema $\begin{array}{ll}\text { mercado virtual } & 168\end{array}$

Figura 52. Diagrama de atividade UTP-C modelado no RSA para o sistema mercado virtual. 


\section{Lista de Tabelas}

Tabela 1. Mapeamentos de informações úteis para coordenação da execução dos testes nos arquivos XML do JAAF+T. 51

Tabela 2. Mapeamento do modelo conceitual para UTP-C. 87

Tabela 3. Perfil dos participantes da avaliação de manutenção. 89

Tabela 4. Informação sobre o sistema de estoque e suprimento de petróleo. 90

Tabela 5. Informação sobre o sistema alocação de petróleo. 91

Tabela 6. Detalhamento dos questionários aplicados. 92

Tabela 7. Perfil das pessoas que participaram da avaliação de criação de modelos. 95

Tabela 8. Tipos de erros identificados nas respostas informadas pelos participantes nas avaliações aplicadas. $\quad 98$

Tabela 9. Mapeamento para transformação de diagramas estruturais da $\begin{array}{ll}\text { UTP-C para o arquivo TF.xml. } & 127\end{array}$

Tabela 10. Mapeamento para transformação de diagramas dinâmicos da $\begin{array}{ll}\text { UTP-C para o arquivo CFF.xml. } & 128\end{array}$

Tabela 11. Análise de quais trabalhos relacionados estão considerando informações de teste manipuladas pela UTP-C. 


\section{Lista de Abreviaturas e Siglas}

$\begin{array}{ll}\text { ISTQB } & \text { International Software Testing Qualifications Board } \\ \text { JAAF+T } & \text { Java self-Adaptive Agent Framework for self-Test } \\ \text { MBT } & \text { Model Based Test } \\ \text { MDD } & \text { Model Driven Development } \\ \text { OMG } & \text { Object Management Group } \\ \text { RQM } & \text { Rational Quality Manager } \\ \text { RSA } & \text { Rational Software Architect } \\ \text { RTM } & \text { Rational TestManager } \\ \text { SMA } & \text { Sistema MultiAgente } \\ \text { SUT } & \text { System Under Test } \\ \text { TMM } & \text { Test Maturity Model } \\ \text { TMMi } & \text { Test Maturity Model integration } \\ \text { UML } & \text { Unified Modeling Language } \\ \text { UTP } & \text { UML Testing Profile } \\ \text { UTP-C } & \text { UML Testing Profile for Coordination }\end{array}$

